\title{
An Assessment of Legibility and Accuracy of Prescriptions through Prescription Surveys
}

\author{
Pragnadyuti Mandal ${ }^{1}$, Swapan Jana ${ }^{2}$, Balaram Ghosh ${ }^{3}$, Souvik Ghosh ${ }^{4}$, Indrani \\ Dalal $^{5} \&$ Kamal Krishna Kundu ${ }^{6}$ \\ ${ }^{1}$ (Department of Pharmacology, Midnapore Medical College, Govt. of West Bengal, India)
}

\begin{abstract}
Aims and Objective: To assess the prescriptions of private practitioners for legibility and accuracy.Materials and Methods: One hundred twenty prescriptions of private practitioners were taken for assessment of legibility and accuracy. The prescriptions were assessed by two investigators and a third adjudicated in case of disagreement. For assessment of handwriting, a scoring method was used. Other parameters which were assessed were: spelling of medicine names, formulation, drugs strength and dosage form, use of nonspecific abbreviations, use of archaic terminologies such as OD, HS etc. and use of capital letters.

Results: Results were expressed in percentages. Spellings of medicine names in all prescriptions were correct. About ninety four percent of prescriptions were legible (scored 3 or 4). In formulations and drugs strength were not mentioned in $4.2 \%$ and $5 \%$ of cases respectively. Abbreviations of names of drugs were written by $6.6 \%$ of prescribers. Sixty percent of prescribers preferred to use archaic terminology and only $6.6 \%$ of the prescribers written the prescriptions in capital letters.

Conclusions: A major percentages (60\%) of private practitioners using the archaic terminologies which usually cause confusion to the dispensers and consumers. Use of capital letter while preparing a prescription, avoiding use of abbreviations and archaic terminologies as well as using the technology such as printing the prescription may ensure a quality service to the consumer.
\end{abstract}

Keywords: prescription, legibility, accuracy.

Submitted date 19 Feb 2013

Accepted Date: 07 Mar 2013

\section{Introduction}

Since the introduction of Consumer Protection Act-1986(CPA-1986) in India, prescription has become a valuable, consumable linkage between the patient and the prescriber. A prescriber, in India, is usually a registered medical practitioner. The ethical and legal duty of a medical practitioner is to write the prescription clearly and legibly [1]. Legibility and accuracy are the essential features of every prescription [2,3].The introduction of 'Right to Information Act 2005'(RTI Act-2005) also extends message to the people about the transparency of any social intervention. Therefore, it is the inherent right of every patient to have a correct, unambiguous and clear prescription. A large number of medical errors include medication errors which may be related to writing of a illegible prescription and dispensing of wrong, inappropriate medications which results in adverse events and death also [2].

There is paucity of data in India in the field of prescription surveys, particularly covering the aspects of legibility and accuracy. But the experts' conclude that ambiguity or confusion in prescription order may be avoided by following some principles while preparing a prescription. These are usually: hand writing-must be clear, spelling of medicine name-must be correct, formulation and strength of medicines-should be quoted clearly, abbreviation of medicine name should be avoided, using archaic terminologies such as Q.D.,O.D.,-should be avoided, leading zero should always be used, capital letter-should be used [2-4].

We made assessment of the prescriptions by following the above parameters which might cause ambiguity in prescription.

\section{Aims and Objectives}

In the background of paucity of literature addressing the prescription related problem in India, this preliminary cross-sectional prescription survey was planned to assess the legibility and accuracy of prescriptions of private practitioners.

\section{Materials and Methods}

For the study, 120 photocopied prescriptions of private practitioners of Kolkata and adjacent areas of West Bengal were collected. From a bunch of nearly1000 prescriptions, 120 prescriptions were collected by 
following the simple random method. The prescriptions were spread on the floor and a ten years old male child was asked to pick up 120 prescriptions one by one with his eyes closed by handkerchief.

In India, a patient usually buys a prescription and it becomes a property and a belonging to him. Therefore, for collecting the prescriptions, written consent from every patient was obtained and all the communications were free from conflicts of interest. The prescriptions were collected from the patients who came to us for counseling about their disease, treatment, drug use, operation and legal problems. The prescriptions of Government and private hospitals were not included for the study. A prescription data record form was prepared. To maintain identity in record each prescription was given a code number starting from 01 to 120 . The prescriptions were assessed by two investigators and a third adjudicated in case of disagreement. The parameters we chose for assessing in every prescription were : Hand writing-assessed by a four point scoring method (Bad=0, $\operatorname{good}=1$, better=2, best=3), Spelling of medicines: correct / incorrect, Formulations and strength of drugs : mentioned/ not mentioned, Abbreviations of drug names: not used/used, Archaic terminology (Q.D., O.D.,H.S., etc ): not used/ used, Leading zero: used/not used, Capital letter: used/ not used [2-4]. Legibility scoring was done by following the scoring method which has been used by others [5]: i) illegible (most or all words impossible to identify), ii) most words illegible; meaning of the whole unclear, iii) some words illegible, but report can be understood by a clinician and iv) legible (all words clear).

\section{Results}

Results were expressed in percentages. Spellings of medicine names in all prescriptions were correct. About ninety four percent of prescriptions were legible (scored 3 or 4 ) and rests of prescriptions were illegible or legible with effort (scored 1 or 2). In formulations and drugs strength not mentioned in the prescriptions were respectively $4.2 \%$ and $5 \%$ of cases. Abbreviations of names of drugs were written by $6.6 \%$ of prescribers. Sixty percent of prescribers preferred to use archaic terminology and only $6.6 \%$ of the prescribers written the prescriptions in capital letters. Nearly sixteen percent of the prescribers did not put the leading zero where applicable.

\section{Discussion}

Illegible prescriptions result in a lower quality of healthcare by loss of time and money, medication errors and patient harm, inefficient or faulty communications and create legal issues [6]. Factors which might affect the aspect and quality of prescribing and errors which may occur while prescribing have been revealed [24, 6]. Remedial measures to avoid the prescription errors and to improve the legibility of prescriptions have also been evaluated by researchers [6]. Legibility of doctors' handwriting was assessed in some studies. In one study, it was found that doctors' handwriting was no less legible than other professionals [7]; in another study, it was revealed that doctors' handwriting when was compared to other healthcare professional and administrators was the worst of all [8].

In this study, we assessed the prescriptions of private practitioners who practice in nursing home or clinic setup. In other studies, the handwritten medication orders or case-notes of indoor and outdoor patients were evaluated. In this study, we observed that $6.6 \%(n=120)$ of prescriptions were illegible or legible with effort and scored 1 or 2 . In two different studies where case-notes $(n=117)$ and out-patient progress notes $(n=50)$ were studied, respectively $15 \%$ and $16 \%$ of the prescriptions were found to be illegible [8,9]. In one study 176 indoor-patients medication orders were evaluated, $20 \%$ of them were illegible or legible with effort [10]. Another study revealed that $15 \%$ of prescriptions of out-door patients were illegible [11]. So far we were unable to relocate any study which would comment about spelling of medicine names. In this study, spellings of medicine names were correct in all prescriptions. In order to get information about the dosage form, formulations-we evaluated about the use of formulations, drugs strength and use of non-specific abbreviations. In $4.2 \%$ and $5 \%$ cases the formulations and drugs strength were not mentioned in the prescriptions, but abbreviations were used in $6.6 \%$ of prescriptions. In one study it was found that $11.4 \%$ of prescribers used wrong drug name, dosage form or abbreviations [12]. Two important factors which usually affects the accuracy of prescriptions and causes confusion and misinterpretation of prescriptions are: use of archaic terminologies such as OD,BD,HS etc. and omission of leading zero where applicable $[4,10]$. In this study, we found that $60 \%$ of the prescribers used archaic terminologies, $15.4 \%$ of prescribers did not put the leading zero and $6.6 \%$ of prescriptions were written in capital letter.

\section{Conclusion}

The study reveals that the private practitioners are not maintaining the standard and are not ensuring adequate quality of prescribing. Generation of a error-free prescription requires adequate care and attention about the multiple components which affects the legibility and accuracy of prescriptions. 
Table-1: Showing the results in percentages:-

\begin{tabular}{|c|c|c|}
\hline Parameters for assessment & & \\
\hline Hand Writing & Score 3 or $4=112(93.4 \%)$ & Score 1 or $2=8(6.6 \%)$ \\
\hline Spelling of medicine name & \multicolumn{2}{|l|}{ Correct in all prescriptions } \\
\hline Formulations & Mentioned in $115(95.8 \%)$ & Not mentioned in $5(4.2 \%)$ \\
\hline Drugs strength & Mentioned in $114(95 \%)$ & Not mentioned in $6(5 \%)$ \\
\hline abbreviations & Used in $8(6.6 \%)$ & Not used in $112(93.4 \%)$ \\
\hline Archaic terminologies & Used in $72(60 \%)$ & Not used in $48(40 \%)$ \\
\hline Leading zero & Used in $11(84.6 \%)$ & Not used in $2(15.4 \%)$ \\
\hline Capital letter & Used in $8(6.6 \%)$ & Not used in $112(93.4 \%)$ \\
\hline
\end{tabular}

\section{REFERENCES}

[1]. WHO Drug action Committee: Model Guide to Good Prescribing; 1995:52

[2]. Ian L.O. Buxton. Principles of Prescription order writing and patient compliance. In Goodman and Gilman's The Pharmacological Basis of Therapeutics. 2006. $11^{\text {th }}$ edition. Macgraw Hill. 1777-1786.

[3]. Lofholm PW, Katzung BG. Rational prescribing and prescription writing. In Basic and Clinical Pharmacology. 2009. $11^{\text {th }}$ edition. Tata Macgraw Hill. 1127-1136.

[4]. Teichman PG., Caffe A.E. Prescription Writing to Maximize Patient Safety. Fam. Pract. Manag. 2002. 9. 27-30.

[5]. Rodriguez-Vera F.J., Marin Y.,Sanchez A., Borrachero C., Pujol E. Illegible handwriting in medical records. J R Soc Med. 2002. 95(11). 545-546.

[6]. Bruner A, Kasdan ML. Handwriting errors: harmful, wasteful and preventable. J Ky Med Assoc. 2001; 99: 189-192.

[7]. Berwick DM, Winikoff DE. The truth about doctors' handwriting: a prospective study. BMJ. 1996; 313(7072): 1657-1658.

[8]. Lyons R, Payne C, McCabe M, et al. Legibility of doctors' handwriting: Quantitative comparative study.BMJ. 1998; $317: 843-844$.

[9]. White KB, Beary JF. Letter to the editor: Illegible handwritten records.N .Engl J Med. 1996; 314(6):390-391.

[10]. Winslow EH, Nestor VA, Davidoff SK, Thompson PG, Borum JC. Legibility and completeness of physicians' handwritten medication orders. Heart Lung. 1997; 26(2): 158-164.

[11]. Meyer TA. Improving the quality of the order-writing process for inpatient orders and outpatient prescriptions. Am J Syst Pharm. 2000; 57 Suppl4: 18-22.

[12]. Lesar TS, Briceland L, Stein DS. Factors related to errors in medication prescribing.JAMA. 1997.277(4): $312-317$ 